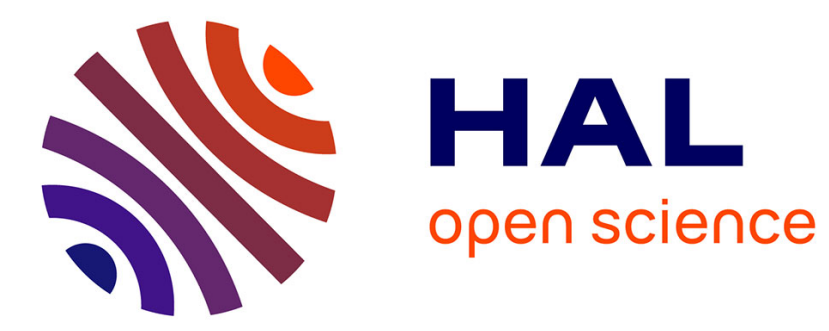

\title{
De la piste animale aux lignes de désir urbaines. Une approche géoichnologique de la trace
}

\author{
Laurent Gagnol, Coralie Mounet, Isabelle Arpin
}

\section{To cite this version:}

Laurent Gagnol, Coralie Mounet, Isabelle Arpin. De la piste animale aux lignes de désir urbaines. Une approche géoichnologique de la trace. L'Information géographique, 2018, 82 (2), pp.11-38. 10.3917/lig.822.0011 . halshs-01875019

\section{HAL Id: halshs-01875019 \\ https://shs.hal.science/halshs-01875019}

Submitted on 3 Sep 2020

HAL is a multi-disciplinary open access archive for the deposit and dissemination of scientific research documents, whether they are published or not. The documents may come from teaching and research institutions in France or abroad, or from public or private research centers.
L'archive ouverte pluridisciplinaire HAL, est destinée au dépôt et à la diffusion de documents scientifiques de niveau recherche, publiés ou non, émanant des établissements d'enseignement et de recherche français ou étrangers, des laboratoires publics ou privés. 


\title{
De la piste animale aux lignes de désir urbaines.
}

\section{Une approche géoichnologique de la trace}

\author{
GAGNOL Laurent, univ. d'Artois, EA Discontinuités \\ laurent.gagnol@univ-artois.fr \\ MOUNET Coralie, univ. Grenoble Alpes, CNRS, Science Po Grenoble*, PACTE, \\ 38000 Grenoble, France, *School of Political Studies \\ coralie.mounet@univ-grenoble-alpes.fr \\ ARPIN Isabelle, univ. Grenoble Alpes, Irstea, UR LESSEM \\ isabelle.arpin@irstea.fr
}

Title : Animal trail and urban desire lines. A geoichnology of the trace.

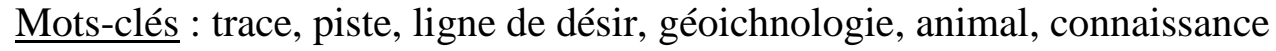

$\underline{\text { Key-words }}$ : trail, track, desire line, geoichnology, animal, knowledge

\section{Résumé :}

S'inscrivant dans le sillage des travaux de Ginzburg sur l'épistémologie des traces et des indices, cet article s'intéresse aux traces et aux connaissances par trace. L'analyse de trois cas d'étude amène à proposer une géographie de la trace (géoichnologie), attentive aux indices, aux cheminements des humains et des non-humains et aux lignes inscrites dans le sol. Ce travail explore tout d'abord les caractéristiques de la trace ainsi que les conditions et les compétences nécessaires au déploiement de savoirs cynégétiques et pastoraux, chez les chasseurs et les naturalistes des Alpes françaises et dans les sociétés pastorales nomades saharo-sahéliennes. Au-delà de ces savoirs particuliers, l'article se penche ensuite sur l'importance des traces dans des espaces plus ordinaires et des pratiques humaines banales. Il montre que les traces des mobilités quotidiennes des habitants de nos villes contemporaines les «lignes de désir »- rendent visibles les manières d'habiter et de circuler en ville, qui diffèrent parfois des mobilités normées par les grands axes et les ordonnancements proposés par l'aménagement urbain. 
«Le navire glisse sur l'eau, le flot fendu se reforme et le sillage s'efface; la terre conserve plus fidèlement la trace des chemins que de bonne heure ont foulés les hommes. La route s'imprime sur le sol ; elle sème des germes de vie : maisons, hameaux, villages, villes. Même ce qui semblerait au premier abord des pistes de hasard, tracées au gré des chasseurs et des bergers, grave son empreinte. Les drailles raient les flancs des Cévennes. À travers les dunes sahariennes, les couloirs (gassi) sont polis par le piétinement des caravanes (...). Ces minces rubans, dont la répétition des pas humains effleure la surface, prétendent déjà à la permanence, revendiquent une personnalité »(P. Vidal de la Blache, 1922).

Comme l'indique cet incipit, l'idée et la notion de trace ont une fonction heuristique ancienne en géographie ${ }^{1}$. Il resterait néanmoins toute une étude à développer concernant la tradition classique ou « vidalienne » en géographie, qui a moins mobilisé le terme de trace que la métaphore de l'empreinte et de l'impression qui se trouve au cœur de la rhétorique explicative de l'inscription des genres de vie dans le paysage ${ }^{2}$ et plus généralement des relations homme-milieu. De la même manière (ou probablement à la suite de quoi), l'idée et la notion de trace ont été essentielles quant aux redéfinitions de l'histoire depuis Langlois et Seignobos (1898), puis dans la première moitié du XXe siècle Bloch (1974) et l'école dite des Annales et, plus tard, chez des auteurs tels que P. Veyne (1979), C. Ginzburg (2010) et Ricœur $(2000)^{3}$.

Si différentes disciplines en sciences humaines et sociales comme en sciences de la vie et de la terre montrent un intérêt renouvelé pour la notion de trace, seuls quelques géographes s'en sont emparés, selon des acceptions différentes. Ainsi, en s'intéressant à l'habiter et aux modes

\footnotetext{
${ }^{1}$ Cette étude sur les connaissances par traces a bénéficié du soutien de la MSHS Lille-Nord de France et de l'Université Grenoble-Alpes.

${ }^{2}$ Cette heuristique de la trace est très présente chez Capot-Rey (1946), géographe spécialiste du Sahara, qui en appelle à une «géographie du mouvement » car « du moment que l'homme qui passe laisse sa trace dans le sol, du moment que ces traces, sollicitant de nouveaux passants, s'enfoncent ou s'élargissent, mais de toute façon deviennent de plus en plus apparentes, on conçoit qu'il puisse y avoir une géographie du mouvement » (p. 18). Les traces creusent dans le paysage un «palimpseste, où les inscriptions des différentes époques se superposent sans s'effacer» (p. 48). Mais son approche classique, écologique et morphologique, tend à privilégier ce qui se conserve et se «sédimente » dans le paysage, autrement dit la trace lorsqu'elle devient une marque stable et durable : «si légère soit la trace d'un pas, elle tend à se perpétuer » (p. 16) et si la piste peut s'évanouir en un «écheveau de fils embrouillés toujours prêts à se rompre» (p. 46), «c'est une chose remarquable que la pérennité de certains itinéraires à travers les siècles» (p. 47). C'est ce qu'il appelle «l'action directrice de la piste »(p. 47). Cette idée est au fondement des études archéogéographiques récentes qui distinguent les grands axes qui perdurent des tracés parfois éphémères qui se réalisent différemment en se déplaçant selon les époques et les moyens de transport : «la multiplicité des traces apparait comme un facteur déterminant dans la résilience des axes sur la longue durée » (Verdier et Robert, 2015, p. 50). Capot-Rey insiste enfin sur la transformation de la trace en piste et en route dans un ordre spatial qui est aussi un ordre d'évolution civilisationnelle : «non seulement la trace se mue en piste, mais la piste elle-même tend à retenir la circulation quand les moyens de transport changent »(Capot-Rey, 1946, p. 16).

${ }^{3}$ Sur les débats concernant la notion de trace en histoire, voir l'interprétation récente de J. Morsel (2016).
} 
d'appropriation mis en lumière et fragilisés par certaines opérations d'urbanisme (renouvellement urbain et patrimonialisation), F. Ripoll (2005) et V. Veschambre (2008) envisagent la trace sous l'angle d'une géographie sociale. B. Steck (2011) mobilise cette notion pour analyser l'organisation et la dynamique des flux (à partir des infrastructures de transport), en insistant sur le rôle essentiel des «portes », qui les canalisent et les captent. Mais c'est l'expression de «traces numériques » qui connait le plus de succès (Serres 2002 ; Galinon-Melenec 2011 ; Mille 2013), ce dont témoigne le grand nombre de publications et de manifestations scientifiques qui l'utilisent sans pour autant le justifier et l'expliciter (par exemple A. Romele et M. Severo, 2015). Le travail de M. Noucher (2015 ; 2017) apporte une grille de lecture innovante en s'intéressant à cette question à partir des évolutions récentes de la fabrique et de l'usage des cartes sous l'influence de la « révolution numérique », analysée également par B. Beaude (2015). Du côté des croisements entre géographie, histoire et archéologie (Snead, Erickson et Darling, 2009), dans le sillage de l'approche archéogéographique développée en France par G. Chouquer, les travaux de S. Robert et N. Verdier $(2009,2015)$ constituent également un jalon important dans la conceptualisation de la notion de trace. Ce tour d'horizon rapide de la mobilisation actuelle de la trace en géographie est sans doute partiel puisque ne sont pas mentionnées les références qui la mobilisent dans leur titre (par exemple Gentelle, 2003) ou dans leur analyse sans pour autant l'ériger en concept heuristique.

Notre travail trouve son origine et son originalité en s'inscrivant résolument dans le sillage des travaux de C. Ginzburg (2010), tout en perpétuant une approche de la trace ancienne en géographie (Capot-Rey, 1946). Précisons d'emblée que nous donnons à la trace une acception précise et restrictive qui la considère avant tout à partir de sa dimension matérielle et spatiale et de sa signification la plus archaïque, au double sens étymologique du terme (commencement/commandement). Dans un texte resté célèbre, C. Ginzburg $(1980 ; 2010)$ envisage une épistémologie des traces et des indices. Il considère que certaines disciplines qualifiées d' « indiciaires » relèvent d'un raisonnement «abductif ». Il fait l'hypothèse que le mode de connaissance qui les sous-tend et les unit est éminemment archaïque et est issu de savoirs ancestraux d'ordre cynégétique. Prenant au sérieux cette hypothèse et sa portée heuristique, nous tentons de saisir les modalités contemporaines de ces savoirs ancestraux communément mobilisés, au sein de milieux et de cultures différents. Contrairement à une conception historienne et philosophique essentiellement fonctionnelle et temporelle (la question de la mémoire chez Ricœur, 2000) ou à une approche sémiotique la considérant 
comme acte performatif (la « rhétorique des cheminements » d'Augoyard, 1979 et la lecture linguistique chez De Certeau, 1980), nous appréhendons la trace à partir de sa manifestation concrète et perceptible par les sens, en tant que produit du passage d'un corps en mouvement (qu'il soit humain ou animal, ce qui peut être généralisé à tout objet et information). Succession d'empreintes de pas (ou de parties du corps), elle représente l'inscription dans le sol et dans le paysage de parcours formés de lignes et de courbes issues de la circulation des êtres vivants et des moyens de transport. Cet article exploratoire prend donc le parti de se déporter du déploiement polysémique et métaphorique actuel de la notion de trace (qu'elles soient numériques, neuronales, psychiques, mnésiques, etc.) pour penser en géographie l'actualisation de son sens archaïque matériel.

Ce travail s'appuie sur nos analyses empiriques concernant la « lecture » des traces animales et humaines chez les sociétés pastorales nomades saharo-sahéliennes (Niger et Tchad) et chez les chasseurs et les naturalistes dans les Alpes françaises. Il explore les caractéristiques de la trace et les conditions et les compétences nécessaires au déploiement de ces savoirs cynégétiques et pastoraux. Mais au-delà des espaces considérés souvent comme naturels et marginaux, au-delà des seules mobilités animales et des seuls spécialistes pastoraux ou cynégétiques des traces, la question des traces concerne aussi des espaces ordinaires facilement accessibles à l'observation et témoigne de pratiques humaines banales mais discrètes. Nous appuyant sur des observations et une analyse exploratoire sur Internet, nous montrons ainsi que les traces des mobilités quotidiennes des habitants de nos villes contemporaines - ce qu'on désigne comme les «chemins de désir » ou «lignes de désir», rendent visibles les manières d'habiter et de circuler en ville, qui diffèrent parfois des mobilités normées par les grands axes et les ordonnancements proposés par l'aménagement urbain. À travers ces cas d'étude, une géographie de la trace (géoichnologie) est donc proposée, attentive aux indices, aux cheminements des humains et des non-humains et aux lignes inscrites dans le sol.

\section{1- Une approche géoichnologique de la trace}

\section{a. La matérialité de la trace}

Cette première partie s'appuie sur deux cas d'études empiriques sur les connaissances par traces exercées dans le pistage et la traque

Le premier cas d'étude porte sur les Alpes françaises et s'intéresse à la manière dont les spécialistes des animaux sauvages (chasseurs, naturalistes) acquièrent des connaissances sur 
ces animaux (loups, sangliers, chevreuils, lièvres, etc.) par les traces qu'ils laissent et qui s'impriment sur des supports tels que la boue, la neige, etc. Les objectifs de ces spécialistes sont multiples : il s'agit de pister l'animal sauvage soit pour parvenir à le voir, à le photographier, à le tuer, soit pour compter les animaux présents sur un territoire, en comprendre le comportement, etc. Ce cas d'étude s'appuie sur des enquêtes par entretiens semi-directifs (une centaine) auprès de chasseurs et naturalistes dans le cadre d'un travail post-doctoral (Irstea) sur les connaissances par traces et de travaux de recherches sur les savoirs cynégétiques (Arpin, Mounet, Granjou, 2014 ; Mounet, Massart, 2017).

Le second cas porte sur les connaissances et savoir-faire relatifs aux traces chez les sociétés pastorales semi-nomades (Niger et Tchad) habitant un milieu désertique et souvent sablonneux favorable à l'enregistrement des empreintes. L'utilité de la «lecture » des traces est quotidienne et consiste à rechercher les animaux domestiques qui divaguent dans les pâturages (dromadaires) ou à retrouver une bête égarée ou volée. Les empreintes des humains sont également examinées, pour retrouver un voleur ou surveiller la population des campements ou du village. Les objectifs de pistage et de traque portent souvent sur des animaux domestiques et des humains que l'on identifie et que l'on connait souvent individuellement (Gagnol, 2013).

L'acception matérielle de la trace nous amène à définir certaines de ses caractéristiques. Les connaissances par traces relevant du pistage reposent en premier lieu sur les empreintes (Chazel et Chazel, 2011) : c'est à partir du négatif du pied, que les spécialistes identifient l'animal (la famille ou l'espèce) ou l'individu qui en est l'auteur. S'imprimant en creux par une pression exercée sur le substrat d'accueil comme la boue, la neige ou le sable, la trace se caractérise par sa nature soustractive (Ingold, 2001), les individus emportant avec eux de la matière provenant du sol. Produit de corps animaux ou humains en mouvement, la trace est ensuite étroitement liée à la mobilité et s'oppose de ce fait à la marque, caractérisée par sa nature ponctuelle et additive. La trace doit dès lors être comprise comme une suite d'empreintes formant une ligne et révélant la circulation d'êtres vivants. L'itinéraire que rend manifeste la trace s'accompagne d'indices de présence et de passage, tels que les déchets ou vestiges, qui sont autant de signes laissés par les êtres vivants (crottes, urines, restes de repas, etc.). Ces indices sont rassemblés et recoupés pour mieux interpréter la trace en elle-même et, in fine, le comportement des individus. 
Le caractère éminemment changeant du substrat (Chazel et Chazel, 2011) participe ensuite au caractère éphémère et labile des traces. Les empreintes et les lignes imprimées au sol s'effacent avec la modification de la qualité du substrat (fonte ou nouvelle chute de neige, lessivage du sol par la pluie, assèchement de la boue, passage du vent dans le sable, etc.). Lors de successions de phases de gel et de dégel, les traces de pas dans la neige sont déformées, agrandies et deviennent plus difficiles à interpréter. Certaines traces, à certains endroits et à certains moments, sont toutefois plus pérennes que d'autres : les empreintes sur la boue sont plus stables que celles imprimées sur une neige potentiellement en fonte par exemple. Mais quelle que soit leur durée de vie, les traces sont vouées à disparaitre en se dégradant progressivement ou soudainement, sauf cas exceptionnels liés à un contexte favorable de fossilisation (en grotte, avant une éruption volcanique, etc.) et qui intéresse la paléoichnologie (sciences des traces fossiles).

La trace est enfin la plupart du temps marquée par sa non intentionnalité : elle est ce qui est laissé involontairement derrière soi, contrairement au marquage intentionnel de l'espace qui s'apparente ici à la marque-déchet-propriété (Serres, 2008). À partir de ces premières caractéristiques de la trace entendue comme ligne d'empreintes, il convient d'explorer plus précisément les spécificités des connaissances par traces, conférées par la matérialité de ces dernières.

\section{b. Une dépendance aux contingences de la production et de l'effacement des traces}

Par le biais des traces, les pasteurs, les chasseurs et les naturalistes disposent de moyens de connaissance indirects et non invasifs des animaux ou des humains (pour)suivis, qui impliquent un rapport au temps spécifique puisque différée dans le temps. Le pisteur engage les trois temporalités du présent, passé et futur. À partir de la reconstitution des évènements passés grâce aux indices présents, il se projette dans le futur pour atteindre un animal ou un humain. Mais ces connaissances sont fragiles car étroitement liées aux caractéristiques propres aux traces, aux contingences de leur existence et de leur évolution.

La «lecture » des traces est tout d'abord liée à la nature du substrat, plus ou moins propice à l'impression de pas. Chez les Touaregs, la plupart des sols constitue un substrat favorable même si le sable mou des dunes ou les sols latéritiques trop indurés ne permettent que difficilement de déceler des empreintes. Chez les pisteurs alpins, la neige ou la boue sont propices mais de façon hétérogène : toutes les qualités de neige ne conviennent pas aussi bien. 
Les neiges dures n'impriment pas, les neiges trop humides déforment les traces de pas, tandis que les neiges trop profondes ne permettent pas une impression nette des contours. Un naturaliste interrogé considère ainsi qu'un seul type de neige constitue un substrat idéal pour le suivi des loups, une couche de neige fraîche de moins de dix centimètres d'épaisseur.

La dépendance des traces au substrat peut provoquer des difficultés de lecture des lignes étudiées, en introduisant une discontinuité liée à la dégradation ou à l'absence soudaine de substrat propice à l'impression. Avec sa capacité à imprimer plusieurs traces, le substrat peut également se transformer en palimpseste: l'interprétation est alors brouillée par les empilements de traces issus de l'activité d'autres êtres vivants. Le passage d'animaux efface les traces préexistantes car «tracer, c'est aussi effacer» (Derrida, 2006), volontairement ou involontairement.

Ensuite, les informations sur lesquelles se fondent ces savoirs sont infimes (Ginzburg, 2010). Le pistage s'appuie sur des observations précises, avec un sens aigu du détail, de telle sorte que les pisteurs s'imaginent parfois à «jouer à Sherlock Holmes » (Eco et Sebeok, 2015) ou "à un jeu de piste », cherchant les menus indices qui demeurent. Insignifiantes pour un non spécialiste, ces traces sont ainsi invisibles ou difficilement repérables pour l'œil non exercé. Elles sont en effet dénuées d'ontologie propre (Ricœur, 2000): les traces sont toujours le signe de quelque chose et nécessitent d'être interprétées et mises en récit pour devenir signifiantes. Elles requièrent un regard attentif et éduqué (Arpin, Mounet, Geoffroy, 2015), pour lequel ces détails anodins constituent une forme d'écriture à déchiffrer : «la neige c'est le livre » explique spontanément un naturaliste, établissant un lien entre ligne et écriture (Derrida, 2006 ; Ingold, 2001). Pour que ces traces prennent un sens, le pisteur doit leur adresser des questions et agencer les réponses qu'il en obtient pour les mettre en intrigue (Ricœur, 2000). Dans la section suivante, nous décrirons les compétences nécessaires à ce déchiffrement et à cette mise en récit.

\section{c. Savoir lire et mettre en récit les traces}

Les connaissances par traces nécessitent un engagement du corps et des sens du pisteur pour repérer ces traces labiles, infimes et difficiles à repérer. Selon Ingold, la perception de l'environnement constitue un processus d'exploration active par un individu en mouvement : l'individu construit son environnement en le parcourant avec son corps et en l'explorant à l'aide de ses sens. Acquérant progressivement des habiletés à percevoir, il est engagé dans un processus continu d' « éducation de l'attention », inextricable de l'expérience ordinaire et de la 
manière d'habiter un lieu (Ingold, 2000). Les connaissances par traces relèvent ainsi d'un rapport spécifique à l'espace : il s'agit d'un savoir sur l'espace et dans l'espace, qui se déploie en situation et s'appuie sur une expérience et une connaissance intime des lieux. Il relève d'un savoir-faire pratique, d'une intelligence de la situation qui rappelle la mètis des grecs incarnée notamment par Hermès (Détienne et Vernant, 1974) et qui a été longtemps glorifié dans les mythes, légendes, contes et poèmes avant d'être conceptualisée (Merton et Barber, 2004) sous le vocable aujourd'hui à la mode de sérendipité (Catelin, 2014)

Le pisteur montre une capacité à repérer des changements infimes dans le milieu ou le territoire connu par le bais d'une acuité et d'une attention visuelles. La perception olfactive peut également être mobilisée avec l'odeur laissée par les individus dans leur sillage ou dans certains de leurs déchets (l'odeur permet par exemple de distinguer les crottes de loups de celles des chiens). L'audition permet aussi de percevoir des indices de présence comme les cris d'animaux. Le pisteur peut enfin «prolonger» ses sens par le biais d'animaux domestiques tels que les chiens dont l'odorat est plus développé : les chasseurs sont accompagnés de chiens «créancés » c'est-à-dire qui réagissent spécifiquement aux pistes olfactives laissées par une espèce de gibier donnée comme le sanglier ou le chevreuil ${ }^{4}$.

Le pistage s'appuie également sur une connaissance corporelle et située du milieu, obtenue au fil du temps par l'arpentage régulier des lieux. S'acquiert progressivement un savoir sur les comportements et les habitudes spatiales des animaux sauvages et domestiques, leur évolution selon la saison ou les conditions météorologiques. Certaines zones a priori favorables à la présence des animaux sont privilégiées pour détecter leurs traces fraîches. Les pasteurs nomades cherchent à anticiper les déplacements de leur bétail à partir de la connaissance de leurs habitudes, du comportement collectif du troupeau ou individuel de chaque animal.

Une fois les traces repérées, leur interprétation et leur mise en récit s'appuient sur un faisceau d'indices recueillis lors du pistage: les traces sont interrogées pour identifier quel(s) individu(s) sont passés, à quel moment et de quelle manière.

Tout d'abord, la lecture des empreintes, des voies et des pistes permet de déduire l'espèce animale voire l'individu, et d'identifier son poids, son âge, son sexe, son état de santé ou encore la présence ou non de bât sur les animaux domestiques. Chez les chasseurs et

\footnotetext{
${ }^{4}$ Cette connaissance par traces est bien entendue partagée avec les animaux dont les capacités sensorielles, dépassant celles des humains, leur permettent le repérage d'autres animaux (d'une même espèce, d'espèces proies ou prédatrices, etc.) mais également d'humains. Un naturaliste interrogé se souvient ainsi que, revenu au point de départ de sa journée après l'avoir passée à chercher des loups, il s'est aperçu que ses traces matinales avaient été recouvertes par celles de loups qui le pistaient...
} 
naturalistes, l'identification est généralement au niveau spécifique mais peut parfois porter sur quelques individus particuliers lorsqu'ils présentent des signes distinctifs. Chez les pasteurs touaregs et toubous, en revanche, l'identification est individuelle, ceux-ci reconnaissant à leurs empreintes chacun des animaux qu'ils possèdent. Fréquemment, ils savent aussi identifier chaque pied de bétail et chaque empreinte des humains du village et des campements alentours. On sait donc lorsqu'on a affaire à des traces étrangères, aussi bien pour les humains (on reconnaît tout de suite l'arrivée d'un « étranger ») que pour les animaux domestiques (il s'agit alors d'animaux perdus ou volés) et même sauvages. La familiarité avec les lieux et l'intimité avec les animaux et les gens se vivent ainsi à travers les traces.

Ensuite, le pisteur peut dénombrer les individus à partir de la lecture des voies. Cela exige souvent patience et ruse. Par exemple, le pistage de loups dans la neige sur des cheminements rectilignes ne permet pas d'identifier leur nombre, ceux-ci marchant à la queue leu leu (selon l'origine étymologique) et il faut attendre un changement de direction pour que les différentes traces se divisent.

Dans la traque, il est important de déterminer le moment auquel est passé l'individu, pour pouvoir éventuellement anticiper le chemin qu'il a parcouru depuis et prédire sa localisation actuelle. Dans le sable, les traces fraîches sont caractérisées par le fait qu'elles n'ont pas été perturbées par le vent et qu'elles n'ont pas été traversées par les insectes nocturnes. Les pasteurs sahariens distinguent l'empreinte neuve qui a quelques heures, de celle qui a une journée et de celle qui a passé une nuit. Dans la neige, les traces fraîches sont caractérisées par leur netteté alors que celles plus anciennes ont subi des transformations du substrat et sont déformées, agrandies ou présentant des contours flous. Les crottes s'effritent ou s'humidifient également au fil du temps. Sur le sol nu, les chiens de chasseurs détectent les pistes olfactives laissées par le gibier et réagissent de manière différente selon la fraicheur de la piste, aboyant ou tirant plus ou moins fort sur la laisse.

Les traces permettent enfin aux pisteurs de conjecturer la manière dont les individus se sont déplacés. Ainsi, les pisteurs sont capables de déduire l'allure de l'animal de la forme des voies tracées dans le sol. Puis, avec l'aide d'autres indices associés à la trace, ils peuvent imaginer son comportement, la survenue d'évènements, voire son intentionnalité : l'animal est-il en train de manger, de se déplacer rapidement ou encore de fuir face à un danger, pourchassé par un prédateur ? Cet extrait d'entretien mené auprès d'un naturaliste montre cette capacité à reconstituer des scènes passées à partir de traces et d'indices : "On trouve une espèce de tunnel dans la neige, on voit des traces d'hermine qui passent dans la neige puis après à 
nouveau un tunnel et des petites traces de sang et puis on voyait arriver des traces de campagnol qui arrivaient. Bon ben on imagine la scène. Hop, l'autre, il s'est fait croquer».

Le but de cette lecture des traces peut être de rattraper ou de retrouver un animal ou un homme comme d'acquérir des connaissances à distance sur les habitudes spatiales des animaux, le nombre d'individus présents, etc. Si l'objectif est de « remonter » des traces, il faut être capable d'anticiper les déplacements de l'individu pisté pour parvenir à le rejoindre. Suivre les traces est alors parfois contre-productif car cela fait perdre un temps précieux lorsque l'on est à la recherche d'un animal égaré qui fait des détours ou d'un voleur qui cherche à faire disparaître les traces ou à les brouiller. C'est pourquoi, pour suivre un animal ou un homme, les pisteurs appliquent ce qu'on pourrait appeler une stratégie navigationnelle (November et al., 2010) ou hodologique ${ }^{5}$. Selon sa connaissance éthologique de l'espèce ou du goût de l'individu, le pisteur se projette dans l'espace et dans le futur pour se rendre directement à la destination finale (un point d'eau, une zone de pâturages, une zone de bauge, etc.). La stratégie hodologique consiste ainsi à se passer au moins momentanément des empreintes ponctuelles, en coupant l'itinéraire supposé formé par celles-ci, pour aller à la rencontre de l'individu par un trajet plus direct et linéaire. Suivre les traces est ainsi un jeu spatial, hodologique précisément, car linéaire et en mouvement.

Cette mise en intrigue et en récit des évènements passés et cette capacité à se projeter dans le temps et l'espace nécessitent un sens et une capacité à se figurer l'intention de l'animal, à explorer le milieu à sa manière et finalement à «devenir animal» (Lorimer, 2008). Elle implique une bonne connaissance des animaux ou des individus mais aussi des lieux où ils évoluent, s'appuyant sur une longue série d'observations des animaux et d'une analyse répétée de leurs traces. Une fois qu'ils pensent avoir atteint l'endroit où se trouve l'animal ou l'humain pisté, le chasseur ou le pasteur peuvent mettre à l'épreuve leur supposition par un principe de bouclage du secteur. Lorsqu'une piste de sanglier entre dans un massif végétal ou forestier, le chasseur en fait le tour pour repérer si l'animal en est sorti ou non. Si aucune trace n'en sort, les chasseurs pratiqueront une battue à cet endroit. Lorsqu'un pasteur suit un animal volé ou des voleurs de bétail, et qu'il les localise dans un village, il procède de la même manière, en vérifiant qu'aucune trace du voleur ou du bétail volé n'en sort (Gagnol, 2013 et, à Madagascar, A. de Saint Sauveur, 2002).

\footnotetext{
${ }^{5}$ L'hodologie (du grec hodos, le chemin), rend compte de la continuité du mouvement et de la linéarité du parcours : l'espace est envisagé en situation de mobilité, comme si le corps était embarqué dans le récit, selon un itinéraire réel ou fictif.
} 
Cette description des usages des traces débouche sur une caractéristique essentielle : ce savoir produit des effets de pouvoir. Il permet de (pour)suivre mais aussi d'identifier et de surveiller le mouvement des êtres, aussi bien humains qu'animaux (Mauz et Granjou, 2010). Il contribue d'une part à la surveillance ou la traque de corps en mouvement (Chamayou, 2010). S'appliquant sur des individus d'une population, il constitue d'autre part un pouvoir individualisant, et plus précisément pastoral comme l'a étudié dans tous les sens du terme Foucault (2001 et 2009). De ce fait, la trace peut être considérée en tant que dispositif potentiel insoupçonné de surveillance des êtres (Gagnol, 2017). Mais la dépendance à une trace éphémère ainsi que la capacité d'échappement des êtres procurent des failles à ces effets de pouvoir. Les humains comme les animaux montrent une capacité à brouiller leur piste. Ces derniers passent par exemple à travers les cours d'eau pour interrompre leur trace. Les manuels de contre-braconnage publiés souvent par dans renégats tout au long des XVIIIe et XIXe siècles exposent les ruses des braconniers et les moyens de les déjouer. Comme l'indique L. Marin (1978), le « récit est un piège » : l'animal peut refermer un piège sur lui en laissant des traces qui seront lues par d'autres mais celui qui les lit peut tout aussi bien se tromper en les déchiffrant.

Enfin, la dépendance à une trace éphémère, qui ne peut être archivée ou qu'il n'y a pas d'intérêt à archiver, confère à ce type de savoir la particularité de se transmettre principalement par l'expérience pratique. C'est ce à quoi remédient aujourd'hui les formes actualisées et technicisées de pistage, avec l'équipement des êtres de dispositifs GPS ou le déchiffrement génétique des déchets laissés par les animaux (excréments, poils, etc.). Si ces savoirs sont étroitement liés à la matérialité de la trace, ils mobilisent des dispositifs d'inscription (Latour, 1985), rejoignant ainsi les disciplines considérées comme galiléennes (Ginzburg, 2010).

\section{d. Voies, pistes, routes}

Si nous avons détaillé les connaissances par traces de type hodologique, s'appuyant sur des lignes éphémères vouées à être effacées, certains peuvent s'incruster dans le paysage par l'usage répété dans le temps. La trace devient alors une marque, reconfigurant plus durablement le territoire. Des drailles dessinées par le trajet des brebis vers (ou depuis) les alpages aux lignes convergeant aux points d'eau sahariens, des pistes des ours sibériens et des bisons de la prairie nord-américaine aux grandes voies de migration des ruminants de la savane africaine, la circulation des animaux sauvages et domestiques zèbre les paysages 
montagnards, steppiques et désertiques. Les chasseurs des Alpes françaises repèrent les « coulées », zones de passage régulier des animaux sauvages se reconnaissant par une légère dépression dans les herbes ou le sol, les «souilles», cuvettes humides et boueuses dans lesquelles les sangliers viennent quotidiennement se rouler pour se débarrasser des parasites et réguler leur température. Enfin, les humains eux-mêmes, par leurs déplacements réguliers parviennent à former des sentiers plus ou moins pérennes. C'est le cas par exemple des chasseurs qui empruntent des sentiers qu'eux seuls pratiquent et connaissent.

Si elles s'inscrivent dans le paysage, ces marques peuvent toutefois varier selon les saisons : le substrat change, depuis la présence d'un manteau neigeux à celle d'un sol nu et les fréquentations des lieux se modifient avec par exemple les migrations saisonnières et altitudinales. La sédimentation des traces conduisant à la marque n'est donc pas un processus irréversible et la marque peut redevenir trace.

Dessinés par l'accumulation de traces individuelles, ces lignes, sentiers, chemins témoignent des pratiques et habitudes spatiales humaines et animales. Mais ils peuvent également indiquer une intentionnalité de marquage du territoire. Certains animaux sauvages comme les loups bornent leur territoire par la présence régulière d'excréments. Luttant contre l'avancée de la végétation, les chasseurs eux-mêmes entretiennent les sentiers qu'ils empruntent. Enfin, le marquage du sol par le piétinement peut constituer une expérimentation esthétique (DidiHuberman, 2008).

À ces sentiers produits par l'usage, caractérisés par leur évolutivité et leur malléabilité, s'opposent les routes goudronnées, les rues et les trottoirs, dont la logique de production repose sur un aménagement durable de gestion des flux. S'ils facilitent et accélèrent leur vitesse, ils contraignent les déplacements en les canalisant. Produits de la modernité, ces formes spatiales additives (des «fils » selon Ingold, 2001) s'affranchissent du substrat et du milieu. Elles ne donnent pas aussi clairement à voir le cheminement des êtres et des objets qui les fréquentent. De manière schématique, on pourrait considérer que les mobilités dans les espaces urbanisés sont conditionnées par ces voies surimposées, tandis que les espaces dits naturels laisseraient libre cours aux sentiers et aux chemins se formant par l'usage et en lien étroit avec le sol et l'environnement en général.

Mais ce n'est pas uniquement par les matériaux constitutifs que l'on différencie la trace de la route. Si la trace repose sur une logique soustractive (érosive) et la route une logique additive (surimposition), ces catégories se brouillent lorsque par exemple les sentiers sont aménagés. 
Dans certains espaces, la trace est considérée comme une menace à endiguer pour éviter l'érosion et l'empiétement sur la végétation. Un sentier non maîtrisé a tendance à se creuser ou se subdiviser en de multiples voies. Il s'agit alors pour les gestionnaires des aires protégées d'apporter des corrections pour empêcher leur prolifération et canaliser les flux de fréquentation dans une sente unique (par le biais de clôtures et de branchages, de zone de végétation dense et d'une signalétique, etc.). Le programme d'action et d'éthique «Leave no trace » (Marion et Reid, 2001 ; Tollis, 2012), initié par des écologistes et gestionnaires étatsuniens, est un principe de gestion et d'éducation à l'environnement sauvage (wilderness) qui pousse au bout cette logique de canalisation des flux touristiques dans les espaces protégés. Il vise un double objectif: limiter l'impact de la fréquentation touristique sur le milieu (dégradation des sols, de la végétation, tranquillité de la faune sauvage, pollution) et préserver le sentiment de solitude au sein de ces espaces en limitant l'étendue de la circulation et des marques de présence humaine (interdiction des feux de camp, obligation de ramener ses déchets dont ses excréments). Dans une logique qui rejoint de ce point de vue les aménagements urbains et les équipements routiers, il s'agit ainsi de transformer des sentierstraces et formés par l'usage en sentiers-routes concentrant l'ensemble de la circulation.

Un autre cas éloigné des précédents nous semble particulièrement intéressant afin de rendre compte de la présence des traces dans les milieux urbains ordinaires : celui des traces appelées « lignes de désir » qui apparaissent spontanément dans les villes à la faveur d'un substrat ou d'un contexte climatique propices.

\section{2- Les lignes de désir : traces de cheminements braconniers en milieu urbain}

La ville est souvent considérée comme un environnement artificiel. Depuis deux ou trois décennies, elle est appréhendée également à partir d'une perspective inverse, en tant que milieu certes marqué par les activités anthropiques mais qui comporte aussi une part de «naturalité ». La nature en ville fait aujourd'hui pleinement partie des approches savantes et des politiques urbaines (Arnould et al., 2011). Si les animaux et les végétaux ont souvent recueilli le plus d'attention, le sol urbain a rarement fait l'objet de réflexions spécifiques (Barles et al., 1999) lorsqu'ils ne relèvent pas de surfaces viabilisées ou jardinées. Pourtant, sur certains lieux et surfaces privilégiés ou dans certains contextes climatiques spécifiques, ils enregistrent et conservent des traces de locomotions ordinaires et d'activités quotidiennes dont ils sont le support. Ces traces sont en soi intéressantes à analyser pour ce qu'elles signifient des pratiques urbaines dans leurs relations voire leurs oppositions avec les aménagements qui 
sont faits en leur nom (de Certeau, 1980). Dans les médias et les réseaux sociaux, mais de plus en plus également en géographie urbaine et en urbanisme, on donne souvent le nom chemins ou de lignes de désir (desirelines ou desirepaths) à ces traces de circulations en milieu urbain.

\section{Lignes d'usages}

Banales et souvent peu visibles, ces traces s'inscrivent dans le sol des terrains meubles des pelouses et des terrains vagues, notamment dans les jardins publics et entre les haies et les parterres de fleurs. Créant parfois de véritables pistes urbaines longues de plusieurs centaines de mètres, elles constituent le plus souvent une simple sente de la longueur de quelques pas à la hauteur de la trouée d'une haie ou un passage qui forme un raccourci en coupant l'angle droit d'un trottoir. Plus ou moins larges et profondes, elles sont creusées par différents types de moyens de circulation qualifiés de «doux » et qui peuvent s'affranchir du réseau routier bitumé (la marche en premier lieu). Ces tronçons ou ces lignes discontinues révèlent des cheminements plus ou moins directs ou sinueux, formant un chenal unique ou multiple et des itinéraires plus ou moins durables. Ils ont tous la particularité d'être créés involontairement, ou plus exactement d'être creusés spontanément, à l'image de la plupart des chemins ruraux vicinaux, par opposition aux rues et aux routes qualifiées de «voies d'Etat» (Verdier et Robert, 2005).

Essentiellement piétonnières, les circulations urbaines douces prennent des trajectoires différentes mais le plus souvent sous forme de courbes que de lignes droites. C'est pourquoi S. Lavadinho (2008) définit la ligne de désir comme «la courbure optimale du tracé qu'un piéton laisse dans son sillage lorsqu'il est totalement libre de son mouvement ». À l'origine cependant, cette expression désignait certes des itinéraires choisis mais à partir d'une approche opposée. Les enquêtes sur le trafic automobile dans les aires métropolitaines américaines dès années 1950 (Weiner, 2016, p. 26), l'ont défini comme une ligne droite abstraite entre deux points (d'origine et de destination). Ces analyses de planification du trafic ont servi à justifier la création de nouvelles infrastructures de transport, notamment autoroutières (Dupuy, 1975). À partir d'enquêtes à domicile, il était demandé aux citadins de sélectionner leurs destinations quotidiennes. P. Claval (1996) précise: «en mettant directement en relation les points d'origine de ces mouvements et le lieu où ils aboutissaient, on obtenait une figuration de la vie de relation à l'intérieur de la ville, indépendamment de le structure des voies. C'est par la comparaison de la carte des lignes de désir (c'est ainsi que les flèches représentant les flux étaient désignées) et de celle des déplacements réels, que les 
ingénieurs pouvaient choisir les itinéraires où faire porter les investissements pour faciliter la circulation globale ». Cela correspond évidemment à une conception géométrique de l'espace, qui réduit le parcours en un trajet en ligne droite parfaite (au sens mathématique, de distance la plus courte entre deux points) au sein d'un espace parfaitement isotrope (Claval, 1981). Aujourd'hui, lorsqu'elles sont appliquées aux traces dans le milieu urbain, les lignes de désir ne sont pas réduites aux chemins les plus courts et directs (L'Hostis, 2016). Elles peuvent correspondre à un itinéraire plus commode et accessible, plus sûr, plus rapide ou plus agréable car à l'écart des nuisances de la circulation routière ou plus proche d'un site plaisant (bord de lac, parcours ombragé, etc.) ou permettant d'éviter un obstacle ou un endroit malaisé à traverser (tunnel, escalier, etc.). Il semblerait que ce sens actuel ne date que des années 1980 lors du réaménagement de Central Park à New York où les nouveaux tracés des chemins ont été fondés sur l'étude des lignes de désir.

\section{Lignes de contestations}

Selon une logique et un but précis, elles sont appelées en anglais des desire lines, ce que les Québécois ont traduit par lignes de désir. Cette expression exprime ainsi la circulation spontanée des citadins, non contrainte par la voirie, les aménagements et le mobilier urbains. Leur intérêt réside aussi dans leur utilisation contestataire. Elles peuvent permettre de mettre en lumière l'inadaptation des aménagements urbains : soit que les trottoirs et les chemins dédiés à la marche sont absents ou discontinus, soit qu'ils sont inadaptés à la circulation piétonne en faisant des détours ou à l'inverse en prenant des formes géométriques (par exemple les carrefours à angle droit). L'apparition spontanée de ces lignes et courbes de désir montrent en creux combien l'aménagement urbain oublie et contraint la circulation douce. Elle pallie ainsi l'absence de trottoirs, de continuité et d'intermodalité. Certains en concluent que les pieds et les traces sont démocratiques et, dans certains cas, contestataires et anarchistes, puisqu'ils s'affranchissent en partie des infrastructures de circulation normée et imposée par les aménageurs. Par les chemins, les citadins non associés aux projets d'urbanisme «votent» avec leurs pieds. L'expression d'urbanisme tactique (tactical urbanism), citoyen et participatif, est mobilisée par des militants qui, au moyen de simples photos et d'un investissement à faible coût, dénoncent les aménagements existants et proposent des améliorations pour les mobilités piétonnes urbaines. Par exemple à Détroit et dans d'autres shrinking cities (Fol S. et Cunningham-Sabot E., 2010), les lignes de désir montrent la déliquescence de la voirie et des investissements publics. Dans des villes 
nouvelles comme Brasilia, elles permettent de critiquer l'urbanisme fonctionnel et le toutautomobile.

Les pouvoirs publics et notamment les services d'urbanisme de nombreuses municipalités intègrent aujourd'hui les lignes de désir à leurs réflexions stratégiques, par exemple en amont d'un réaménagement des circulations et du mobilier dans un parc. Certaines municipalités choisissent de les stabiliser pour qu'elles deviennent de véritables voies piétonnes ou cyclables viabilisées en posant des dalles par exemple. Mais elles sont souvent considérées comme relevant de pratiques braconnières de l'espace et font l'objet de politiques répressives ou correctives pour les supprimer, les limiter ou empêcher leurs apparitions spontanées. Des barrières sont érigées et une signalétique appropriée les signale même si, bien souvent, elles réapparaissent ailleurs.

\section{Sneckdown ou l'aménagement 2.0}

Prenons enfin l'exemple de ces lignes de désir lorsque la ville est recouverte de neige. Le passage répété des piétons, cyclistes, automobilistes laisse des traces puis des chemins en tassant ou faisant fondre la neige, aidé en cela par le salage, tandis que les lieux non fréquentés conservent la neige plus longuement. La neige agit comme un papier calque montrant les pratiques spatiales «libres » et moins contraintes des citadins puisque la neige dissimule une partie des aménagements urbains. La neige montre notamment les voies désirées des piétons, tandis que l'emprise spatiale de la circulation automobile est considérablement réduite. Elle permet ainsi de redistribuer l'ordre de la circulation et la hiérarchie des usages, voire de favoriser des pratiques ludiques insolites ou dissidentes (Bérard, 2016).

La route s'affine pour les voitures et le trottoir s'étale un peu plus pour les piétons. Ce phénomène s'observe notamment au niveau des intersections. La neige qui redessine la rue montre en creux combien les axes routiers et notamment les carrefours empiètent sur l'espace public, les lieux de stationnement et de circulation des piétons et de celles et ceux qui optent pour un autre mode de circulation. La neige en resserrant la voierie effectivement utilisée permet en outre de réduire la distance des passages piétons au niveau des carrefours et de calmer le trafic en ralentissant la vitesse. Des îlots sont créés ainsi que des avancées de trottoirs par extension des bordures des routes. Par ce gain d'espace, d'autres possibilités d'aménagements sont ouvertes en valorisant d'autres usages. 
C'est pourquoi des photographes et des urbanistes « amateurs » cherchent à fixer par l'image (photographies et vidéos) ces états des lieux éphémères, instantanés des mouvements dans la ville. Leur objectif critique est de montrer à quel point l'urbanisme est pensé avant tout par et pour l'automobile. Certains vont jusqu'à proposer d'autres types d'aménagement en redessinant l'espace public par une retouche des photographies (infographie et cartographie). Cette pratique photographique et de planification urbaine participative par la neige s'appelle en anglais sneckdown, mot composé de snowy (enneigé) et de neckdown (avancées de trottoirs) ${ }^{6}$. S'il n'y a aucune trace de pneus dans certaines zones enneigées, les automobilistes en ont-ils réellement besoin pour assurer la fluidité de leurs déplacements quotidiens ? N'y at-il pas moyen de mieux rentabiliser l'espace public et d'en rendre une partie aux piétons ?

Les pouvoirs publics ont là encore deux attitudes. Soit ils font appel via les réseaux sociaux aux adeptes des sneckdown, soit ils opposent des arguments contraires : resserrer la voirie réduirait le nombre de places de parkings et poserait problème aux services de sécurité (ambulance, police, pompiers, etc.).

Les lignes de désir et les traces urbaines, tout en faisant l'objet d'une appropriation habitante et artistique renouvelée, commencent à entrer dans les pratiques des gestionnaires et des urbanistes, mais sans que pour l'instant elles soient reconnues comme un outil opérationnel généralisable et fassent l'objet d'une construction théorique. D'ailleurs, l'expression de lignes de désir devient courante et son sens s'élargit, en s'articulant notamment avec l'idée de dépendance au sentier dans le monde des outils numériques et même en sociologie puisque des chercheurs évoquent des social desirepaths. Dans une étude récente sur la ville postApartheid, elles désignent l'écart entre l'espace vécu et l'espace planifié ${ }^{7}$. Leur intérêt réside dans la façon dont elles donnent à voir la manière dont les êtres habitent leur ville et participent à la fabrication environnementale. Elles révèlent des enjeux insoupçonnés de la

\footnotetext{
${ }^{6}$ Le terme «sneckdown » a été proposé sur Tweeter le 2 janvier 2014 à 23h19 (heure de New York) par un bloggeur et urbaniste Aaron Naparstek. Mais l'idée a été documentée sous la forme d'un film dès 2011 par un réalisateur, Clarence Eckerson Jr., qui a popularisé le postage de vidéos et de photos à ce sujet. À la suite d'une période de blizzard à New York en 2011, il a observé que le rétrécissement de la chaussée par la neige, qui canalise les voitures, allonge les coins de rue à l'image des neckdown qui diminuent la chaussée pour inciter les passants à traverser au niveau des passages piétons. Il a même écrit l'histoire du mot et de l'idée sur son blog Streetfilms.org. Depuis 2014 le mot-clé est très présent dans les réseaux sociaux et les plates-formes de dépôt de photos et de vidéos.

7 « space between the planned and the providential, the engineered and the « lived », and between official projects of capture and containment and the popular energies which subvert, bypass, supersede and evade them » (Murray et al., 2007, p. 1).
} 
trace et des connaissances par traces dans des contextes ordinaires et quotidiens. En outre, elles soulèvent la question de la capacité contestataire des traces face à un agencement spatial imposé et parfois contraignant.

Arnould P., Le Lay Y., Dodane C. et Méliani I. (2011), « La nature en ville : l'improbable biodiversité », Géographie, économie, société, vol. 13, pp. 45-68.

Arpin I., Mounet C. et Geoffroy D. (2015), «Inventaires naturalistes et rééducation de l'attention », Études rurales, $\mathrm{n}^{\circ} 195$, pp. 89 - 108.

Arpin I., Mounet C. et Granjou C. (2014), « Jusqu'où explorer les relations entre humains et animaux ? », in V. Despret et R. Larrère (dir.), Les animaux : deux ou trois choses que nous savons d'eux, Hermann Ed., coll. «Colloque de Cerisy », pp. 253-267.

Augoyard J.-F. (1979), Pas à pas. Essai sur le cheminement quotidien en milieu urbain. Paris, Seuil, 190 p.

Barles S., Breysse D., Guillerme A. et Leyval C. (1999), Le sol urbain. Paris, Anthropos, $278 \mathrm{p}$.

Beaude B. (2015), «Spatialités algorithmiques », in M. Severo et A. Romele (dir.), Traces numériques et territoires, Paris, Presses des Mines, pp. 133-160.

Bérard R. (2016), « Du tourisme inattendu ? Quand la neige s'invite en ville », Via@, 1(9), https://viatourismreview.com/fr/2016/10/quand-la-neige-sinvite-en-ville/

Bloch M. (1974 [1941/42]), Apologie pour l'histoire ou Métier d'historien, Paris, Armand Colin, $110 \mathrm{p}$.

Capot-Rey R. (1946), Géographie de la circulation sur les continents, Paris, Gallimard, 296 p.

Catelin C. (2014), Sérendipité. Du conte au concept, Paris, Seuil, 270 p.

Certeau (de) M. (1980), L'Invention du quotidien, 1. : Arts de faire, Paris, Gallimard, $416 \mathrm{p}$.

Chamayou G. (2010), Les chasses à l'homme. Histoire et philosophie du pouvoir cynégétique, Ed. La fabrique, 225 p.

Chazel M., Chazel L. (2011), Reconnaitre et décoder les traces d'animaux. Manuel d'ichnologie, Ed. Quae, 190 p.

Claval P. (1981), La logique des villes. Essai d'urbanologie. LITEC, coll. «Géographie économique et sociale », Paris, 633 p. 
Claval P. (1996), La géographie comme genre de vie : un itinéraire intellectuel, Paris, L'Harmattan, $196 \mathrm{p}$.

Derrida J. (2006), L'animal que donc je suis, Paris, Ed. Galilée, 232 p.

Détienne M., Vernant J.-P. (1974), Les ruses de l'intelligence. La mètis des Grecs, Paris, Flammarion, 317 p.

Didi-Huberman G. (2008), La ressemblance par contact. Archéologie, anachronisme et modernité de l'empreinte, Paris, Ed. de minuit, 379 p.

Eckerson C. Jr. (2014), The Complete Origin of the \#Sneckdown, mis en ligne le 6 février 2014 [http://www.streetfilms.org/the-complete-origin-of-the-sneckdown/]

Eco U., Sebeok T. A. (dir.), (2015 [1984]), Le signe des trois. Dupin, Holmes, Pierce, Presses universitaires de Liège, $276 \mathrm{p}$.

Fol S., Cunningham-Sabot E. (2010), « «Déclin urbain » et shrinking Cities : une évaluation critique des approches de la décroissance urbaine ». Annales de géographie, 674, (4), pp. 359-383.

Foucault M., (2001 [1981]), « «Omnes et singulatim »: vers une critique de ma raison politique », in Dits et Ecrits, t. II, Gallimard, pp. 953-980.

Foucault M. (2009), Sécurité, territoire, population, Cours au collège de France, 1977-1978, Gallimard-Seuil, 430 p.

Gagnol L. (2013), «Identifier, rechercher et surveiller par les traces. Eléments d'analyse d'un savoir-faire pastoral chez les sociétés saharo-sahéliennes », in Techniques et culture, $\mathrm{n}^{\circ} 61$ «Vivre le sable! », pp. 166-187.

Gagnol L. (2017), «Les frontières nomades : de la ligne à la trace », in F. Moullé (dir.), Frontières, Presses universitaires de Bordeaux, pp. 183-205.

Galinon-Mélénec B. (dir.) (2011), L’Homme-trace. Perspectives anthropologiques des traces contemporaines, CNRS Ed., 410 p.

Gentelle P. (2003), Traces d'eau. Un géographe chez les archéologues. Paris, Belin, $239 \mathrm{p}$.

Ginzburg C. ([1979] 2010), « Traces. Racines d'un paradigme indiciaire », in Mythes, emblèmes, traces : morphologie et histoire. Nouvelle édition augmentée. Paris, VerdierPoche, $376 \mathrm{p}$.

Ingold T. (2000), The perception of the environment. Essays in livelihood, dwelling and skills. London, New York, Routledge

Ingold T. (2001), Une brève histoire des lignes, Paris, Ed. Zones sensibles, 251 p. 
Langlois C.-V., Seignobos C. (1898), Introduction aux études historiques, Paris, Hachette.

Latour B. (1985), «Les « vues» de l'esprit. Une introduction à l'anthropologie des sciences et des techniques », Culture technique, ${ }^{\circ} 14$, pp. 5-29.

Lavadinho S. (2008), « Chemins de traverse et lignes de désir », in Dossier : « Marcher », Revue Urbanisme ${ }^{\circ} 359$, mars/avril.

L'Hostis A. (2016), « Detour and break optimising distance, a new perspective on transport and urbanism », Environment and Planning B : Urban Analytics and City Science,, vol. 44, Issue 3, pp. 441 - 463.

Lorimer J. (2008), « Couting corncarkes: the affective science of the UK corncrake census », Social studies of science, vol.38, n³, pp. 377-405.

Marin L. (1978), Le récit est un piège, Paris, Ed. de Minuit, 152 p.

Marion J. L., Reid S. E. (2001), «Development of the U.S. Leave No Trace Program : An Historical Perspective », In M. Usher, ed. Enjoyment and Understanding of the Natural Heritage. Scottish Natural Heritage, Edinburgh, The Stationery Office Ltd., Scotland, pp. 8192.

Merton R.K., Barber E. (2004), The Travels and Adventures of Serendipity: A Study in Sociological Semantics and the Sociology of Science, Princeton University Press, Princeton and Oxford, $313 \mathrm{p}$.

Mille A. (2013), « Des traces à l'ère du Web », Intellectica, 59-1, pp. 7-28.

Mauz I., Granjou C. (2010), «Biodiversité et technicisation du suivi des animaux sauvages », in J.-P. Moriceau et P. Madeline (dir.), Repenser le sauvage grâce au retour du loup. Les sciences humaines interpellées, coéd. Presses universitaires de Caen et MRSH, pp. 159-170.

Morsel J. (2016), « Traces? Quelles traces? Réflexions pour une histoire non passéiste », Revue historique, 2016/4, pp. 813-868.

Mounet C., Massart C. (2017), «Contradiction autour de la (dé)légitimation de l'engagement corporel. Pratiques cynégétiques et naturalistes ». Presses des Mines. Actes Quatrièmes ateliers sur la contradiction, ASLC 2016, Expérience, expertise et expérimentation, pp. $61-70$.

Murray N., Shepherd N. et Hall M. (2007), Desire Lines: Space, Memory and Identity in the Post-Apartheid City, New-York, Routledge, 328 p. 
Noucher M. (2015), « De la trace à la carte et de la carte à la trace : pour une approche critique renouvelée des nouvelles sources de fabrique cartographique », in $\mathrm{M}$. Severo (ed.), Traces numériques et territoires, Paris, Presses des Mines, pp. 213-223.

Noucher M. (2017), Les petites cartes du web. Approche critique des nouvelles fabriques cartographiques, Paris, Éd. Rue d'Ulm/Presses de l'École normale supérieure, $65 \mathrm{p}$.

November V., Camacho-Hübner E. et Latour B. (2010), «Entering a risky territory : space in age of digital navigation », Environment and Planning D : Society and Space, vol. 28 , pp. $581-599$.

Ricœur P. (2000), La mémoire, l'histoire, l'oubli. Paris, Seuil, 675 p.

Ripoll F. (2005), «Réflexions sur les rapports entre marquage et appropriation de l'espace », in T. Bulot et V. Veschambre (dir), Mots, Traces et Marques, Paris, l'Harmattan, pp. 15-36

Robert S., Verdier N. (2009), «Pour une recherche sur les routes, voies et réseaux ». Les Nouvelles de l'archéologie, Maison des Sciences de l'Homme, pp.5-8 ; 53-56.

Saint-Sauveur A. (de) (2002), «Prévention des vols de bétail chez les agropasteurs bara de Madagascar. Une conception défensive du patrimoine » in M.-C. Cormier-Salem, D. Juhé-Beaulaton, J.-B. Boutrais, B. Roussel (eds), Patrimonialiser la nature tropicale. Dynamiques locales, enjeux internationaux, Paris, IRD, pp. 245-258.

Serres A. (2002), «Problématiques de la trace à l'heure du numérique », SensDessous, 2012/1 ( $\left.{ }^{\circ} 10\right)$, pp. 84-94.

Serres M. (2008), Le mal propre. Polluer pour s'approprier ?, Paris, Ed. Le Pommier, $112 \mathrm{p}$

Severo M., Romele A. (dir.) (2015), Traces numériques et territoires, Paris, Presses des Mines, $270 \mathrm{p}$.

Snead J.E., Erickson L. et Darling J.A. (2009), Landscapes of Movement : Trails, Paths and Roads in Anthropological perspective, University of Pennsylvania Press, 364 p.

Steck B. (2011), «Flux et territoires : de la trace à la marque, une question de distance », in B. Galinon-Melenec (éd.), L’Homme trace. Perspectives anthropologiques des traces contemporaines, Paris, CNRS Editions, pp 249-270

Tollis C. (2012). Bien gérer les «espaces de nature », une éthique du faire avec: propositions pour une géographie des Associations hétérogènes. Thèse de doctorat de sciences du territoire. Université de Grenoble. 
Verdier N., Robert S. (2015), «Route, réseau, chevelu : une archéogéographie des lignes et des traces », Carnets du Paysage, $\mathrm{n}^{\circ} 27, \mathrm{pp} .42-55$.

Veschambre V. (2008), Traces et mémoires urbaines, enjeux sociaux de la patrimonialisation et de la démolition, Rennes, Presses universitaires de Rennes, $315 \mathrm{p}$

Veyne P. (1979), Comment on écrit l'histoire ; suivi de "Foucault révolutionne l'histoire », Paris, Seuil, 242 p.

Vidal de la Blache P. (1922), Principes de Géographie humaine, Paris, Armand Colin, 362 p.

Weiner E. (2016), Urban transportation Planning in the United States. History, Policy and Practice, 5e Ed., Springer, 442 p. 